\title{
Horn Clause Techniques in Rehabilitation Clinic for Return to Work
}

\author{
Rawia Mohamed- Najeeb Abu Amr, Waidah Ismail, Lailatul Qadri Zakaria, Nurzi Juana Mohd Zaizi, \\ Roesnita Ismail, Mahadi Bahari, Maryati Mohd. Yusof
}

\begin{abstract}
Rehabilitation therapy aims to enable disabled patients to maintain optimal functioning upon achieving returnto-work (RTW) approval. The Berg Balance Scale (BBS) is a benchmark for activity accomplishment featured in the International Classification of Functioning, Disability and Health Framework (ICF). The ICF is used by physiotherapist (PT) and occupational therapist (OT) to determine the functional mobility of disabled patient. Conventionally, practitioners measure, record, and analyze assessment results manually which resulting in difficulty in predicting patient progression. The large data volume involved in the $R T W$ process requires synthesize and reasoning for decision making purposes. Thus, there is a need to identify an efficient reasoning technique to facilitate the decision making process. This study highlights the use of Horn Clause in decision tree to assist medical doctor in assessing improvement of RTW approved patient based on the BBS assessment. The Horn Clause is one of the fundamental reasoning techniques applied in various domains including healthcare. The next phase of this study is to test the efficiency of the Horn Clause reasoning based on patient's stroke recovery and spinal cord injury.
\end{abstract}

Index Terms: Berg Balance Scale (BBS), Decision tree, Horns Clause, Rehabilitation therapy, Return-to-work (RTW).

\section{INTRODUCTION}

Rehabilitation therapy aims to enable disabled patients to maintain optimal functioning upon achieving return-to-work (RTW) approval. During rehabilitation assessment, physiotherapists (PT) and occupational therapists (OT) normally employed the Berg Balance Scale (BBS) as a benchmark for activity accomplishment featured in International Classification of Functioning, Disability and Health Framework (ICF). PT and OT can determine the functional mobility of disabled patients easily based on ICF to help the disabled patient to achieve the goal for rehabilitation therapy.

Revised Manuscript Received on June 22, 2019.

Rawia Mohamed- Najeeb Abu amr, Faculty of Science and Technology, Universiti Sains Islam Malaysia (USIM).

Waidah Ismail, Faculty of Science and Technology, Universiti Sains Islam Malaysia (USIM), Malaysia. Green Science and Technology Research Centre, Islamic Science Institute, Universiti Sains Islam Malaysia (USIM), Malaysia

Lailatul Qadri Zakaria, Faculty of Information Science \& Technology, Universiti Kebangsaan Malaysia, Malaysia

Nurzi Juana Mohd Zaizi, Faculty of Science and Technology, Universiti Sains Islam Malaysia (USIM), Malaysia

Roesnita Ismail, Faculty of Science and Technology, Universiti Sains Islam Malaysia (USIM), Malaysia

Mahadi Bahari, Faculty of Computing, Universiti Kebangsaan Malaysia, Malaysia

Maryati Mohd. Yusof, Faculty of Information Science \& Technology, Universiti Kebangsaan Malaysia, Malaysia
The main goal of rehabilitation process is to improve the physical and functional abilities of disabled patients. This is usually carried out through daily exercises performed regularly at certain time depending on the affected part of the body [1-2]. During early recovery, PT usually administers repetitive exercises that are essential in motion recovery process. The repetition exercises allow simultaneous improvement in the affected muscles and joints [3]. Each patient shows different ability of movement known as Range of Motion (ROM). According to Perkeso, [4] the success RTW program should give the RTW approval for the patient to return to same job same employer, similar job same employer, different job same employer, same job different employer, different job different employer or to do self-employed job[4].

RTW program involved numerous processes, specific features, and complicated relationships between them, which increases the complexity, creates high-dimensionality problems, and difficulty of making decision for the disabled patient. Ming et al. [5]

Large data volume involved in RTW process requires synthesize and reasoning. Thus, there is a need for a system that applies logical roles to this large data volume. These rules will be applied to deduce an assessment for the patient who suffers from disability to give consultation upon achieving return-to-work (RTW) approval. Therefore, there is a need to identify an efficient reasoning technique to facilitate the decision making process. In clinical decision support systems, there are many proposed techniques for decision making process. In addition, decision tree is a common technique used in data mining while Horn Clause is one of the fundamental reasoning techniques applied in various domains including healthcare. Applying Horn Clause in the decision tree could possibly assist practitioners to predict the progression of the disabled patients based on the BBS assessment. The Horn Clause will be developed based on experts in the Rehabilitation Center. The main objective of this paper is to propose a new reasoning technique that uses Horn Clause to support decision making in RTW assessment.

\section{LITERATURE REVIEW}

The development of decision hierarchies in engineering domain is based on drawing mechanism. This development proved that the hierarchies and data mining techniques are useful for classification of the features in complex relationships.

\section{Published By:}

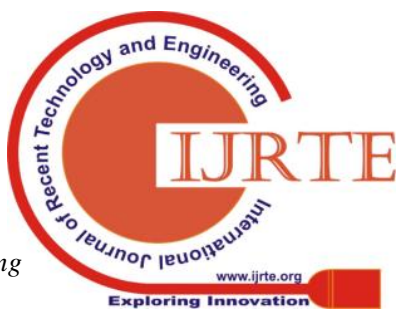


In the field of rehabilitation assessments, it often involved large and complex data and relationships. Ontology can be used to represent, transfer and reuse information and knowledge between users and software in different domains including rehabilitation in healthcare [6-7].

An ontological approach can consolidate knowledge from the existing literature, providing a solid knowledge for specific domain based for tool construction. Ontologies have emerged as an alternative to databases in applications that require a more 'enriched' meaning due to their capability to improve the performance of large data volume [8]. This is because the ontologies break a lot of unnecessary complexity that exist in the large traditional data base[9]. For example, Thuan et al. [10] proposed an ontology-based decision tool that supports business process. On the other hand, ontologies have been used in healthcare field to automatically share and reuse knowledge in CDSSs. This facilitates the use of knowledge to provide evidence-based and personalized therapies in addition of evaluating the effectiveness of a treatment. There are much standard ontology used for this purpose such as and not as a limitation ICF, ICD and SNOMED CT [11].

For rehabilitation purpose, ontologies have been used in many studies to represent person's medical information. For example, robles-bykbaev et al, [12] proposed an expert system able to automatically infer general intervention guidelines for children with disabilities and communication disorders. The system relies on ontologies and implements a semantic web environment to provide several services related with information querying, reports generation, inference of intervention strategies, etc. Moreover, Subirats and Ceccaroni, [13] proposed ontology based decision support system for rehabilitating people with functional diversity. One more example of ontology model which developed for an Upper Limb stroke rehabilitation known as 'ULSOnt' is illustrated in Figure 1. This Ontology enables Semantic knowledge representation of PIS [14]. The part of models includes diagnosis, score ranking and range of movement assessment are defined as following :

a. Diagnosis: The patients will be diagnosis with their stroke subtype (i.e. intracerebral hemorrhage, or subarachnoid hemorrhage).

b. Score ranking: The assessment includes Barthel Index, Lawton Scale, Hospital Anxiety Depress Scale (HADS), Functional Ambulation (FAC), Frenchay Athesia Test (FAsT), Mini-mental State Exam (MmSE), and Caregiver Strain Index (CSI) assessments.

c. Range of movement assessment: The range of movement assessments includes Active Range of Movement (AROM), Passive Range of Movement (PROM), and Muscle Strength Grade at Active Range of Movement (MSG AROM).

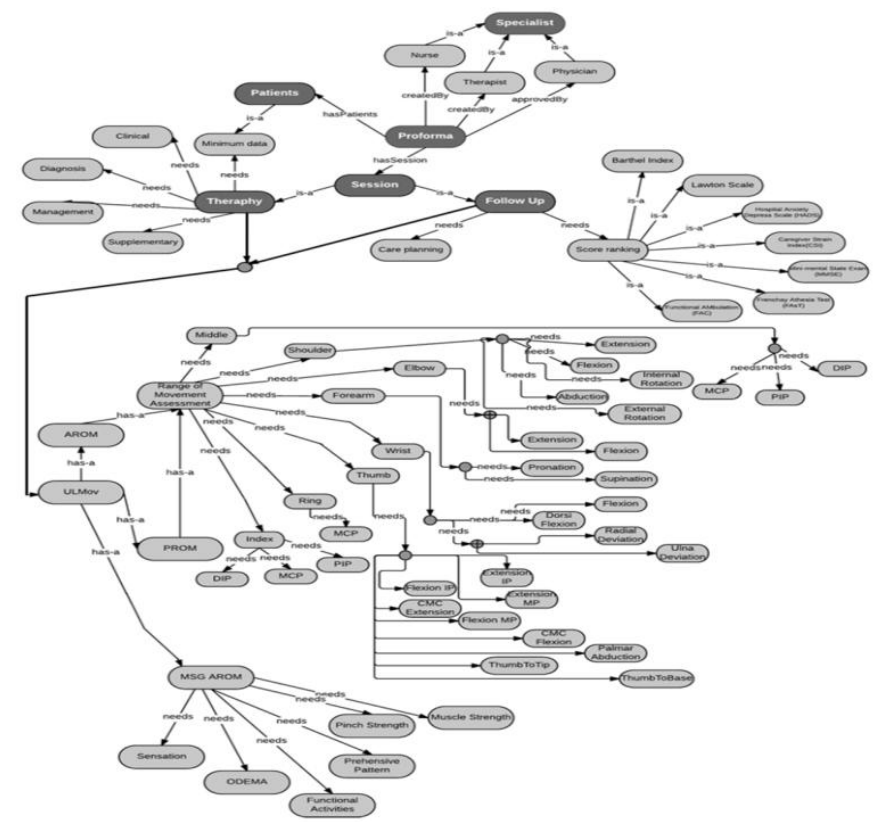

Fig. 1 ULSOnt Ontology (adapted from Afandi et al. [14])

In Developing a CDSS, the main purpose is to find the most accurate and timely medical diagnoses or evaluation using the knowledge that was introduced to the system through ontology (knowledge base). This purpose is achieved in the reasoning part of the CDSS. There are many methodologies have been used for reasoning which applied in CDSS such as Fuzzy reasoning rule-based reasoning case-based reasoning [15]. For example Ping et al. [16] used multiple measurement case-based reasoning (MMCBR) in producing a model that can help in treating liver disease which achieved performance better than classical CBR. On the other hand, the CBR is integrated with a multi agent system to design a CDSS which explore gastric cancer [17]. Furthermore, Wang et al. [18] presented an adaptive neurofuzzy reasoning system (ANFIS) which achieves an accurate system for treating esophageal cancer patients. The reasoning methodologies are applied through different techniques such as and not limited forward chaining, backward chaining and rule elicitation. These techniques used to describe what the problem is, rather than a procedure or algorithm stating how to solve the problem [19-20]. For solving the problem described in reasoning techniques, declarative programming languages which based on mathematical functions and/or logic are clever enough to figure out how to solve the problem. Prolog is an example for declarative programming languages which based on the Horn Clause subset of first order logic. Prolog is perhaps the most prominent of these declarative languages [20].

Horn clauses are a restricted kind of formula in propositional or first-order logic which are computationally tractable, yet highly expressive. Thus, The Horn Clause is one of the fundamental reasoning declarative languages applied in various domains including healthcare [21]. Aliqbal, [21] used horn clause as a fundamental reasoning technique to proposed a web based CDSS that served the rural medical centers. 
It is important to mention that horn clause has not been used in rehabilitation field before. Thus, this study explores the applicability of the Horn Clause in decision tree to assist practitioners in predicting the progression of the disabled patients based on the BBS assessment. The BBS has always been used to objectively assess whether a disabled patient with impairment can balance safely over a series of predetermined tasks. It is a valid instrument used for evaluating patients suffering from Spinal Cord Injury and Stoke Recovery.

\section{METHODOLOGY}

The main feature of BBS knowledge-based is the horn clause rules defined according to the ROM and implemented in a decision tree. This instrument could potentially aids physician to examine patients during RTW decision making and subsequent therapy arrangement. The integration of ontology-based knowledge with decision tree plays an important role in predicting patient progression. The sample of the ROM rules is illustrated in Table 1.

Table 1. ROM for the shoulder

\begin{tabular}{|c|c|c|}
\hline Joint & $\begin{array}{l}\text { Range of } \\
\text { motion }\end{array}$ & Rules \\
\hline$\frac{\bar{d}}{\frac{\bar{d}}{\overrightarrow{0}}}$ & Abduction & $\begin{array}{l}x<45^{\circ}=0, \\
45^{\circ}<x<90^{\circ}=1, \\
90^{\circ}<x<150^{\circ}=2, \\
x>150^{\circ}=3\end{array}$ \\
\hline
\end{tabular}

Figure 2 illustrates how a specialist observed ROM to asses the motion ability of a patient's upper limb. As shown in Table 1, the rules essentially guide specialist to observe the patient's ROM.

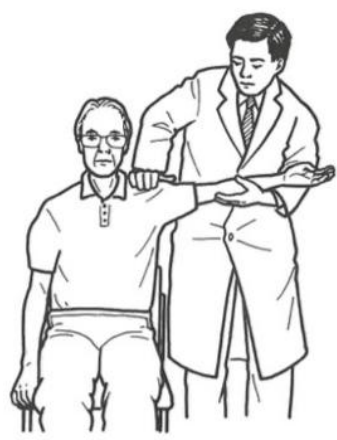

Fig. 2 Assessment of shoulder ROM [11].

\section{THEORY}

For instance, when passive shoulder abduction is limited to less than $45^{\circ}$, a score of 0 is given to a particular patient. Similarly, score 1 means that the joint can be abducted from $45^{\circ}$ to $90^{\circ}$; score 2 indicates abduction from $90^{\circ}$ to $150^{\circ}$; and score 3 indicates that abduction of the shoulder beyond $150^{\circ}$ is possible [10]. Based on the scoring of BBS, the decision tree designed and categorized into three interpretations. These interpretations are high fall risk, medium fall risk and low fall risk. Figure 3 depicts a sample of a decision tree designed based on the BBS. Furthermore, figure 3 show that the decision will be Redo Treatment (RT) when the interpretation is high fall risk, while the BBs assessment recommended to be repeated when the interpretation is medium risk fall. On the other hand the patient achieved the RTW goal if his score indicates that he is under low fall risk interpretation. Table 2 depicts the instances that will be created and trained in the proposed decision tree.

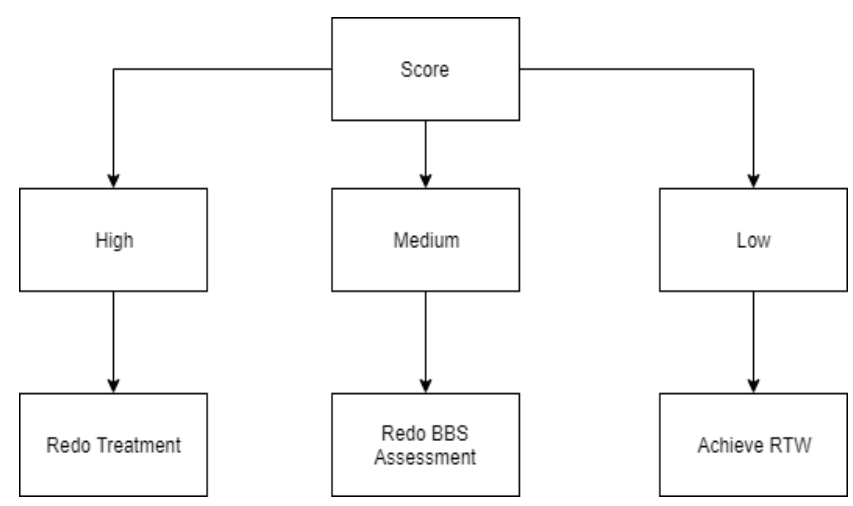

Fig. 3 Decision tree of Berg Balance Scale.

\section{Table 2. List of instances for decision tree with the} category.

\begin{tabular}{|l|l|l|}
\hline $\begin{array}{l}\text { Scorin } \\
\mathrm{g}\end{array}$ & $\begin{array}{l}\text { Interpretatio } \\
\mathrm{n}\end{array}$ & Decision \\
\hline $\begin{array}{l}0<\mathrm{X} \leq \\
20\end{array}$ & $\begin{array}{l}\text { High fall } \\
\text { risk }\end{array}$ & $\begin{array}{l}\text { Redo the } \\
\text { treatment }\end{array}$ \\
\hline $\begin{array}{l}21<\mathrm{X} \\
\leq 40\end{array}$ & $\begin{array}{l}\text { Medium fall } \\
\text { risk }\end{array}$ & $\begin{array}{l}\text { Redo the } \\
\mathrm{BBS} \\
\text { assessmen } \\
\mathrm{t}\end{array}$ \\
\hline $\begin{array}{l}41<\mathrm{X} \\
\leq 57\end{array}$ & Low fall risk & $\begin{array}{l}\text { Achieve } \\
\text { RTW }\end{array}$ \\
\hline
\end{tabular}

\section{ANALYSIS}

It is very important to enhance the decision based on the diagnosis. Thus, the decision is enhanced using horn clause for two types on diagnosis which are stroke Recovery (SR) and Spinal Cord Injury (SCI). For example, the score of the BBS between 0 and 20 means that the interpretation is high fall risk, but this risk may be caused by SR or SCI. Thus, the decision will be affected and related to the diagnosis. Where there are two probabilities of decisions for each diagnosis. As shown in table 3 for the patient scored from 0 to 20 and has been diagnosed as SR may be recommended to do NT or RT. On the other hand, the same two decisions can be recommended for the patients who have been diagnosed as SCI. In terms of achieve RTW decision, it can be recommended when the score of the BBS between 41 to 57 which indicates that the interpretation is low fall risk either for the SR or SCI diagnosis as shown in horn clause equation 9 and 12 and in Figure 4. 
Table. 3 List of Enhancement Based on The Diagnosis

\begin{tabular}{|c|c|c|c|}
\hline Scoring & Interpretation & Diagnosis & Decision \\
\hline $0<X \leq 20$ & High fall risk & $\begin{array}{l}\text { Stroke } \\
\text { recovery }\end{array}$ & $\begin{array}{l}\text { New } \\
\text { treatment }\end{array}$ \\
\hline $0<X \leq 20$ & High fall risk & $\begin{array}{l}\text { Stroke } \\
\text { recovery }\end{array}$ & $\begin{array}{l}\text { Redo } \\
\text { treatment }\end{array}$ \\
\hline $0<X \leq 20$ & High fall risk & $\begin{array}{l}\text { Spinal } \\
\text { Cord } \\
\text { Injury }\end{array}$ & $\begin{array}{l}\text { New } \\
\text { treatment }\end{array}$ \\
\hline $0<X \leq 20$ & High fall risk & $\begin{array}{l}\text { Spinal } \\
\text { Cord } \\
\text { Injury }\end{array}$ & $\begin{array}{l}\text { Redo } \\
\text { treatment }\end{array}$ \\
\hline $21<X \leq 40$ & $\begin{array}{l}\text { Medium fall } \\
\text { risk }\end{array}$ & $\begin{array}{l}\text { Stroke } \\
\text { recovery }\end{array}$ & $\begin{array}{l}\text { New } \\
\text { treatment }\end{array}$ \\
\hline $21<\mathrm{X} \leq 40$ & $\begin{array}{l}\text { Medium fall } \\
\text { risk }\end{array}$ & $\begin{array}{l}\text { Stroke } \\
\text { recovery }\end{array}$ & $\begin{array}{l}\text { Redo } \\
\text { treatment }\end{array}$ \\
\hline $21<X \leq 40$ & $\begin{array}{l}\text { Medium fall } \\
\text { risk }\end{array}$ & $\begin{array}{l}\text { Spinal } \\
\text { Cord } \\
\text { Injury }\end{array}$ & $\begin{array}{l}\text { New } \\
\text { treatment }\end{array}$ \\
\hline $21<\mathrm{X} \leq 40$ & $\begin{array}{l}\text { Medium fall } \\
\text { risk }\end{array}$ & $\begin{array}{l}\text { Spinal } \\
\text { Cord } \\
\text { Injury }\end{array}$ & $\begin{array}{l}\text { Redo } \\
\text { treatment }\end{array}$ \\
\hline $41<X \leq 57$ & Low fall risk & $\begin{array}{l}\text { Stroke } \\
\text { recovery }\end{array}$ & $\begin{array}{l}\text { Redo } \\
\text { treatment }\end{array}$ \\
\hline $41<X \leq 57$ & Low fall risk & $\begin{array}{l}\text { Stroke } \\
\text { recovery }\end{array}$ & $\begin{array}{l}\text { Achieve } \\
\text { RTW }\end{array}$ \\
\hline $41<X \leq 57$ & Low fall risk & $\begin{array}{l}\text { Spinal } \\
\text { Cord } \\
\text { Injury }\end{array}$ & $\begin{array}{l}\text { Redo } \\
\text { treatment }\end{array}$ \\
\hline $41<X \leq 57$ & Low fall risk & $\begin{array}{l}\text { Spinal } \\
\text { Cord } \\
\text { Injury }\end{array}$ & $\begin{array}{l}\text { Achieve } \\
\text { RTW }\end{array}$ \\
\hline
\end{tabular}

An example of Horn Clause rules that can be created based on the decision tree are as follows:

$$
\begin{aligned}
& \neg \mathrm{H} \cap \neg \mathrm{SR} \cap \mathrm{NT} \\
& \neg \mathrm{H} \cap \neg \mathrm{SR} \cap \mathrm{RT} \\
& \neg \mathrm{H} \cap \neg \mathrm{SCI} \cap \mathrm{NT} \\
& \neg \mathrm{H} \cap \neg \mathrm{SCI} \cap \mathrm{RT} \\
& \neg \mathrm{M} \cap \neg \mathrm{SR} \cap \mathrm{NT} \\
& \neg \mathrm{M} \cap \neg \mathrm{SR} \cap \mathrm{RT} \\
& \neg \mathrm{M} \cap \neg \mathrm{SCI} \cap \mathrm{NT} \\
& \neg \mathrm{M} \cap \neg \mathrm{SCI} \cap \mathrm{RT} \\
& \neg \mathrm{L} \cap \neg \mathrm{SR} \cap \mathrm{RTW} \\
& \neg \mathrm{L} \cap \neg \mathrm{SR} \cap \mathrm{RT} \\
& \neg \mathrm{L} \cap \neg \mathrm{SCI} \cap \mathrm{RTW} \\
& \neg \mathrm{L} \cap \neg \mathrm{SCi} \cap \mathrm{RT}
\end{aligned}
$$

\section{RESULTS AND DISCUSSION}

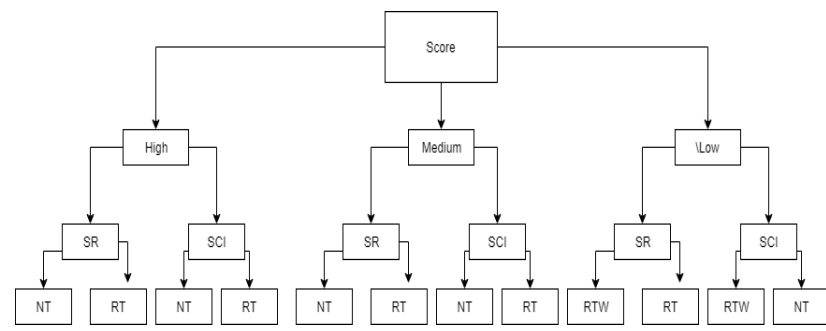

Fig. 4. Decision tree enhancement based on Table 3.

\section{CONCLUSION}

The rehabilitation therapy needs computerized techniques which help PT and OT in assisting patient while conserving effort and time. This study explores the applicability of the Horn Clause technique in predicting the progression of disabled patients based on the BBS assessment. In the current practice, practitioners at rehabilitation clinic make decision manually to approve RTW patients. Since this process involved a large volume of data, particularly those of exercise assessments, it is time consuming and cumbersome. Therefore, there is a need to identify an efficient reasoning technique to facilitate this process. This study proposed the use of Horn Clause in assisting clinicians in assessing improvement and recommending therapy for RTW patients. In order to test its efficiency, the Horn Clause technique need to be validated by using patients' data. For example, stroke recovery and spinal cord injury.

\section{ACKNOWLEDGEMENTS}

The authors would like to acknowledge the supporters of this project which is a part by Fundamental Research Grant Scheme FRGS, Ministry of Higher Education, University Sains Islam Malaysia (USIM), Faculty of Science and Technology (FST), Project code(USIM/FRGS/FST/055002/51918).

\section{REFERENCES}

1. Olaleye O.A., Hamzat T. K., Owolabi M. O.: Stroke rehabilitation: should physiotherapy intervention be provided at a primary health care centre or the patients place of domicile? Disability and Rehabilitation. 36, 49-54 (2014).

2. Teasell, R., Meyer M. J., McClure, A., Pan, C., Murie-Fernandez, M., Foley, N., Salter, K. Stroke rehabilitation: an international perspective. Topics in Stroke Rehabilitation. 16, 44-56 (2009).

3. Arredondo, R., Garcia, D. A., Morris, M., Tosunoglu, S.: A review of rehabilitation strategies for stroke recovery. ASME Early Career Technical Journal. 11, 24-31(2012).

4. https://www.perkeso.gov.my/index.php/en/accordion-b/programreturn-to-work,2016, (accessed 2019).

5. Ming, Z., Wang, G., Yan, Y., Panchal, J. H. Goh, C. H., Allen, J. K., Mistree, F.: Ontology-Based Representation of Design Decision Hierarchies. Journal of Computing and Information Science in Engineering. (2017). doi: 10.1115/1.4037934.

6. Roussey, C., Pinet, F., Kang, M. Corcho, O.: An Introduction to Ontologies and Ontology Engineering Catherine. In Falquet, G., Métral, C., Teller, J., Tweed, C. (pnyt). Ontologies in Urban Development Projects. pp. 9-38. London: Springer-Verlag (2011). 
7. Vasconcelos, J. B., Kimble, C., Rocha, Á.: Competence management in knowledge intensive organizations using consensual knowledge and ontologies. Information Systems Frontiers. 18(6). doi: 10.1007/s10796016-9627-0. (2016)

8. Abas, H. I., Yusof, M. M., Mohd Noah, S. A.: The application of ontology in a clinical decision support system for acute postoperative pain management. In: International Conference on Semantic Technology and Information Retrieval, STAIR 2011.pp. 106-112 (2011).

9. M. Uschold, "Ontology and database schema: What' $\mathrm{s}$ the difference ?," vol. 10, pp. 243-258, 2015.

10. Thuan, N. H. Antunes, P., Johnstone, D.: A Decision Tool for Business Process Crowdsourcing: Ontology, Design, and Evaluation. Group Decision and Negotiation. pp. 1-28 (2018).

11. L. Subirats and L. Ceccaroni, "An ontology for computer-based decision support in rehabilitation," in Lecture Notes in Computer Science (including subseries Lecture Notes in Artificial Intelligence and Lecture Notes in Bioinformatics), 2011.

12. V. E. Robles-bykbaev, D. Quisi-peralta, and U. Polit, "An ontologybased expert system to generate therapy plans for children with disabilities and communication disorders," 2016.

13. L. Subirats and L. Ceccaroni, "An ontology for computer-based decision support in rehabilitation," in Lecture Notes in Computer Science (including subseries Lecture Notes in Artificial Intelligence and Lecture Notes in Bioinformatics), 2011.

14. Afandi, R. R., Radman, A., Bahari, M., Zakaria, L. Q., Mustapha, M., Ismail, W.: Ontology Development in Patients Information System for Stroke Rehabilitation. In: 8th International Conference on Biomedical Ontology (2017).

15. N. Shoaip, S. El-sappagh, S. Barakat, and M. Elmogy, Chapter 4 Reasoning methodologies in clinical decision support systems: A literature review. Elsevier Inc., 2019.

16. X.-O. Ping, Y.-J. Tseng, Y.-P. Lin, H.-J. Chiu, F. Lai, J.-D. Liang, G.T. Huang, P.-M. Yang, A multiple measurements case-based reasoning method for predicting recurrent status of liver cancer patients, Comput. Ind. 69 (2015) 12-21.

17. Y. Shen, J. Colloc, A. Jacquet-Andrieu, K. Lei, Emerging medical informatics with casebased reasoning for aiding clinical decision in multi-agent system, J. Biomed. Inform. 56 (2015) 307-317.

18. C.-Y. Wang, J.-T. Tsai, C.-H. Fang, T.-F. Lee, J.-H. Chou, Predicting survival of individual patients with esophageal cancer by adaptive neuro-fuzzy inference system approach, Appl. Soft Comput. 35 (2015) 583-590.

19. J. D. Summers, "DETC2005-85334 REASONING IN ENGINEERING DESIGN," no. January 2005.

20. T. F. Gordon and H. Friedrich, "Representing Argumentation Schemes with Constraint Handling Rules ( CHR )." Canadian Social Sciences and Research Council (SSHRC) in the Carneades project, by Microsoft, in the DUCK project, and by the European Union Horizon 2020 research and innovation programme under grant agreement No 732189 in the AEGIS project.2018.

21. R. A. I. Iqbal, "Hybrid Clinical Decision Support System: An Automated Diagnostic System For Rural,” 2012. 